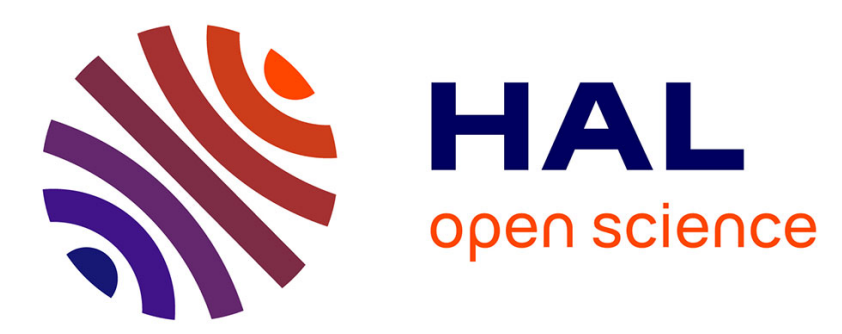

\title{
Energy-Efficient Trajectory Generation for a Hexarotor with Dual-Tilting Propellers
}

Fabio Morbidi, Davide Bicego, Markus Ryll, Antonio Franchi

\section{To cite this version:}

Fabio Morbidi, Davide Bicego, Markus Ryll, Antonio Franchi. Energy-Efficient Trajectory Generation for a Hexarotor with Dual-Tilting Propellers. IEEE/RSJ International Conference on Intelligent Robots and Systems (IROS 2018), Oct 2018, Madrid, Spain. pp.6226-6232, 10.1109/IROS.2018.8594419 . hal-01891460

\section{HAL Id: hal-01891460 \\ https://hal.science/hal-01891460}

Submitted on 9 Oct 2018

HAL is a multi-disciplinary open access archive for the deposit and dissemination of scientific research documents, whether they are published or not. The documents may come from teaching and research institutions in France or abroad, or from public or private research centers.
L'archive ouverte pluridisciplinaire HAL, est destinée au dépôt et à la diffusion de documents scientifiques de niveau recherche, publiés ou non, émanant des établissements d'enseignement et de recherche français ou étrangers, des laboratoires publics ou privés. 


\title{
Energy-Efficient Trajectory Generation for a Hexarotor with Dual-Tilting Propellers
}

\author{
Fabio Morbidi ${ }^{1}$, Davide Bicego ${ }^{2}$, Markus Ryll ${ }^{3}$, Antonio Franchi ${ }^{2}$
}

\begin{abstract}
In this paper, we consider a non-conventional hexarotor whose propellers can be simultaneously tilted about two orthogonal axes: in this way, its underactuation degree can be easily adapted to the task at hand. For a given tilt profile, the minimum-energy trajectory between two prescribed boundary states is explicitly determined by solving an optimal control problem with respect to the angular accelerations of the six brushless motors. We also perform, for the first time, a systematic study of the singularities of the control allocation matrix of the hexarotor, showing the presence of subtle singular configurations that should be carefully avoided in the design phase. Numerical experiments conducted with the FAST-Hex platform illustrate the theory and delineate the pros and cons of dual-tilting paradigm in terms of maneuverability and energy efficiency.
\end{abstract}

\section{INTRODUCTION}

\section{A. Motivation and related work}

In the last decade, electrically-powered rotary-wing Unmanned Aerial Vehicles (UAVs) have experienced a dramatic diffusion. Their maneuverability and small launch-andlanding footprint have made them very popular, but their aerodynamics have some disadvantages, such as the relatively poor energy efficiency. Moreover, existing lithium-ion polymer (LiPo) batteries have a high energy-to-weight ratio and a high-rate discharge capability [1], but their reduced capacity is major issue, limiting de facto flight endurance to 15 to 30 minutes, thus precluding utilization in persistent missions. Several solutions contributing towards increased endurance of quadrotors have recently appeared in the literature. To overcome the problem of fast depletion of LiPo batteries, several companies (Intelligent Energy, EnergyOr, etc.) have developed electrical quadrotors powered by hydrogen fuel cells. This technology holds great promise since flight endurance can be extended up to two and a half hours, and the vehicle can be refueled almost instantly after landing. However, some concerns remain about safe handling of hydrogen indoors and about payload reduction. Perching/climbing systems [2], [3] and more efficient rotor configurations [4] have also been explored for quadrotors. Other studies have envisaged to extend UAV's mission time by dumping exhausted battery modules out of the aircraft in flight, thus reducing its mass [5]. Aerial robots tethered to a ground station have also gained prominence in the last

\footnotetext{
${ }^{1}$ Université de Picardie Jules Verne, MIS laboratory, 80039 Amiens, France, fabio.morbidi@u-picardie.fr

${ }^{2}$ LAAS-CNRS, Université de Toulouse, CNRS, 31400 Toulouse, France, davide.bicego@laas.fr, antonio.franchi@laas.fr

${ }^{3}$ MIT, Computer Science and Artificial Intelligence Laboratory, Cambridge, MA 02139, USA, ryllemit.edu

This work has been partially funded by the European Union's Horizon 2020 research and innovation program under grant agreement No 644271 AEROARMS.
}

few years. In fact, the tether can be used to provide energy to the UAV, thus offering virtually unlimited flight time [6]. Abdilla et al. [7] have characterized the energy consumption of a rotorcraft powered by LiPo batteries in hovering flight and introduced an endurance estimation model tailored to the Parrot AR.Drone 2.0. Similarly, in [7], in [8] an analytical framework has been presented for predicting the hovering time. Finally, wireless power transfer systems have been developed in [9], [10] to minimize down time for recharging or replacing batteries and enable longer flight times.

The problem of generating energy-optimal trajectories for a rotorcraft has received much less attention in the aerial robotics literature. In [11], an energy-efficient pathplanning strategy has been proposed for a hexarotor on a multi-target mission. However, an approximated energy cost function which does not explicitly account for the physical parameters of the electrical motors is considered. In [12], the authors have studied the relationship between navigation speed and energy saving for the simplified kinematic model of a quadrotor following an assigned trajectory. A model of the LiPo battery of the Parrot AR.Drone 2.0 was identified, but as in [11], the specifications of the electrical motors were not considered for estimating energy consumption.

\section{B. Original contributions, organization and notation}

This paper builds upon our previous work on energy management for quadrotor UAVs [13]. In [13], we obtained minimum-energy trajectories between two prescribed boundary states by solving an optimal control problem with respect to the angular accelerations of the four brushless DC (BLDC) motors. Inspired by [14], [15], in this work we extend this formulation to a hexarotor whose propellers can be simultaneously tilted about two orthogonal axes via two servomotors (henceforth referred to as dual-tilting propellers). When compared to the complex design of the existing omnidirectional aerial robots with eight tilted reversible motors [16], [17], the dual-tilting mechanism offers greater flexibility since the underactuation degree of the vehicle can be easily adapted to the specific task at hand [14]. In particular, the hexarotor can be reconfigured in forward flight to behave as a fully-actuated platform and is capable of independently generating thrust and torque in any direction, which simplifies the execution of physical interaction tasks with the environment [18]. This attractive morphologyadaptation capability partially compensates for increased vehicle mass and extra mechanical/control complexity. In view of the tilt-planner design, we also provide, for the first time, a complete characterization of the singularities of the control allocation matrix of the hexarotor. Finally, to validate the 
proposed theory and study the interplay between maneuverability and energy efficiency, we numerically compute minimum-energy trajectories for the FAST-Hex platform [14] in realistic scenarios, using the TOMLAB Optimization Environment [19]. The rest of this paper is organized as follows. In Sect. II, we present the dynamic model of the hexarotor with dual-tilting propellers and perform the singularity analysis. In Sect. III, we introduce the electrical model of a BLDC motor and formulate the minimum-energy trajectory generation problem. The results of numerical experiments are discussed in Sect. IV, and some possible directions for future research are presented in Sect. V.

Notation: Throughout this paper, $\mathbb{Z}$ denotes the set of integers, $\mathbb{R}^{n}$ the $n$-dimensional Euclidean space, $\mathbf{I}_{n}$ the $n \times n$ identity matrix, $\mathbf{0}_{n \times m}$ the $n \times m$ matrix of zeros, and $\boldsymbol{e}_{i}$ the $i$ th element of the standard basis of $\mathbb{R}^{3}$. Moreover, $\mathrm{SO}(3)$ indicates the special orthogonal group in dimension three, and $\mathbf{R}_{z}(\theta), \mathbf{R}_{y}(\theta)$ and $\mathbf{R}_{x}(\theta)$, the $3 \times 3$ elementary rotations of an angle $\theta$ about the $z-, y$ - and $x$-axis, respectively.

\section{HeXARotor with DUAL-TILTING PROPELLERS}

\section{A. Dynamic model}

Let $\mathcal{F}_{\mathrm{W}}=\left\{O_{\mathrm{W}} ; \mathbf{x}_{\mathrm{W}}, \mathbf{y}_{\mathrm{W}}, \mathbf{z}_{\mathrm{W}}\right\}$ be the world inertial frame, $\mathcal{F}_{B}=\left\{O_{B} ; \mathbf{x}_{B}, \mathbf{y}_{B}, \mathbf{z}_{B}\right\}$ the body frame rigidly attached to the hexarotor (where $O_{B}$ corresponds to the geometric center of the six propellers and to the center of mass, $\mathrm{CoM}$, of the platform), and let $\mathcal{F}_{S_{i}}=\left\{O_{S_{i}} ; \mathbf{x}_{S_{i}}, \mathbf{y}_{S_{i}}, \mathbf{z}_{S_{i}}\right\}$, $i \in\{1, \ldots, 6\}$, be the frame associated to propeller $i$. The origin $O_{S_{i}}$ coincides with the CoM of motor $i$, the rotation plane of propeller $i$ is parallel to the plane defined by the axes $\mathbf{x}_{S_{i}}$ and $\mathbf{y}_{S_{i}}$, and $\mathbf{z}_{S_{i}}$ is the spinning axis of the propeller along which the thrust force is generated. We will assume that propeller $i$ can be tilted of an angle $\alpha$ about the $\mathbf{x}_{S_{i}}$-axis and of an angle $\beta$ about the $\mathbf{y}_{S_{i}}$-axis ${ }^{1}$ (see Fig. 1). A tilt planner controls two servomotors and a transmission system to synchronize the angle $\alpha$ and $\beta$ of the six propellers (see Sect. II-B for some design considerations). Following [14], [15], the equations of motion of the hexarotor can be written as:

$\left[\begin{array}{c}m \ddot{\mathbf{p}}_{B} \\ \mathbb{J} \dot{\boldsymbol{\omega}}_{B}\end{array}\right]=-\left[\begin{array}{c}m g \boldsymbol{e}_{3} \\ \boldsymbol{\omega}_{B} \times \mathbb{J} \boldsymbol{\omega}_{B}\end{array}\right]+\left[\begin{array}{cc}\mathbf{R}_{B} & \mathbf{0}_{3 \times 3} \\ \mathbf{0}_{3 \times 3} & \mathbf{I}_{3}\end{array}\right]\left[\begin{array}{l}\mathbf{F}_{1}(\alpha, \beta) \\ \mathbf{F}_{2}(\alpha, \beta)\end{array}\right] \boldsymbol{u}$,

where $\mathbf{p}_{B}=[x, y, z]^{T}$ denotes the position of $O_{B}$ with respect to $\mathcal{F}_{\mathrm{W}}, \mathbf{R}_{B} \in \mathrm{SO}(3)$ represents the orientation of $\mathcal{F}_{B}$ with respect to $\mathcal{F}_{\mathrm{W}}$, and $\boldsymbol{\omega}_{B} \in \mathbb{R}^{3}$ is the angular velocity of $\mathcal{F}_{B}$ with respect to $\mathcal{F}_{\mathrm{W}}$, expressed in $\mathcal{F}_{B}$. Finally, $m$ denotes the total mass of the hexarotor, $\mathbb{J} \in \mathbb{R}^{3 \times 3}$ the inertia matrix of the rigid body with respect to $O_{B}$ expressed in $\mathcal{F}_{B}, g=9.8066 \mathrm{~m} / \mathrm{s}^{2}$ the acceleration due to gravity, and $\boldsymbol{u}=\left[f_{1}, \ldots, f_{6}\right]^{T}$ where $f_{i}$ is the magnitude of the force generated by propeller $i$. For the control allocation matrices $\mathbf{F}_{1}, \mathbf{F}_{2} \in \mathbb{R}^{3 \times 6}$ in (1), we have that [14]:

$\sum_{i=1}^{6} \mathbf{f}_{i}^{B}\left(f_{i}, \alpha, \beta\right)=\mathbf{F}_{1}(\alpha, \beta) \boldsymbol{u}$,

$\sum_{i=1}^{6}\left(\mathbf{p}_{B, S_{i}}^{B} \times \mathbf{f}_{i}^{B}\left(f_{i}, \alpha, \beta\right)\right)+\boldsymbol{\tau}_{i}^{B}\left(f_{i}, \alpha, \beta\right)=\mathbf{F}_{2}(\alpha, \beta) \boldsymbol{u}$,

\footnotetext{
${ }^{1}$ For the sake of simplicity, in the sequel the distance between the origin $O_{S_{i}}$ and the center of propeller $i$ will be neglected.
}

where, for $i \in\{1, \ldots, 6\}$,

$$
\begin{aligned}
\mathbf{f}_{i}^{B}\left(f_{i}, \alpha, \beta\right) & =f_{i} \mathbf{R}_{S_{i}}^{B}(\alpha, \beta) \boldsymbol{e}_{3}, \\
\boldsymbol{\tau}_{i}^{B}\left(f_{i}, \alpha, \beta\right) & =(-1)^{i-1} c_{f}^{\tau} f_{i} \mathbf{R}_{S_{i}}^{B}(\alpha, \beta) \boldsymbol{e}_{3},
\end{aligned}
$$

are the thrust force and drag torque, respectively, and

$$
\begin{aligned}
& \mathbf{p}_{B, S_{i}}^{B}=\ell \mathbf{R}_{z}((i-1) \pi / 3) \boldsymbol{e}_{1}, \\
& \mathbf{R}_{S_{i}}^{B}(\alpha, \beta)=\mathbf{R}_{z}((i-1) \pi / 3) \mathbf{R}_{x}\left((-1)^{i-1} \alpha\right) \mathbf{R}_{y}(\beta),
\end{aligned}
$$

where $\mathbf{R}_{S_{i}}^{B}(\alpha, \beta) \in \mathbf{S O}(3)$ represents the orientation of $\mathcal{F}_{S_{i}}$ with respect to $\mathcal{F}_{B}, \ell>0$ is the distance between $O_{B}$ and $O_{S_{i}}$, and $c_{f}^{\tau}>0$ (meters) is a constant parameter which depends on propeller geometry and profile (see Fig. 1). Note that the alternating sign in (3b) models the fact that propellers with adjacent indices are designed to spin in opposite directions and thus generate opposite drags. Moreover, in order to guarantee full actuation of the platform, in (4b) we have assumed that the propellers with adjacent indices tilt (inward/outward) in opposite directions of an angle $\alpha$ about the $\mathbf{x}_{S_{i}}$-axis.

\section{B. Singularity analysis and tilt-planner design}

Let us define:

$$
\mathbf{F}(\alpha, \beta)=\left[\begin{array}{l}
\mathbf{F}_{1}(\alpha, \beta) \\
\mathbf{F}_{2}(\alpha, \beta)
\end{array}\right] \in \mathbb{R}^{6 \times 6} .
$$

Note that since $\left[\begin{array}{cc}\mathbf{R}_{B} & \mathbf{0}_{3 \times 3} \\ \mathbf{0}_{3 \times 3} & \mathbf{I}_{3}\end{array}\right]$ has full rank, then $\operatorname{rank}\left(\left[\begin{array}{cc}\mathbf{R}_{B} & \mathbf{0}_{3 \times 3} \\ \mathbf{0}_{3 \times 3} & \mathbf{I}_{3}\end{array}\right] \mathbf{F}(\alpha, \beta)\right)=\operatorname{rank}(\mathbf{F}(\alpha, \beta))$. To study the input singularities of system (1), we can then limit ourselves to the control allocation matrix $\mathbf{F}(\alpha, \beta)$ which maps the input force vector $\boldsymbol{u}$ onto a control wrench (for a general classification of rotary-wing UAVs based on the dimension of the freely-assignable input force subspace, see [15, Sect. III]). These singularities have an impact on the optimal trajectory-generation problem studied in Sect. III-B: hence, we will find below all values of $\alpha$ and $\beta$ for which $\mathbf{F}(\alpha, \beta)$ becomes rank deficient. Using (2), we can obtain explicit expressions for $\mathbf{F}_{1}(\alpha, \beta)$ and $\mathbf{F}_{2}(\alpha, \beta)$, leading to the $\mathbf{F}(\alpha, \beta)$ reported in (5), where,

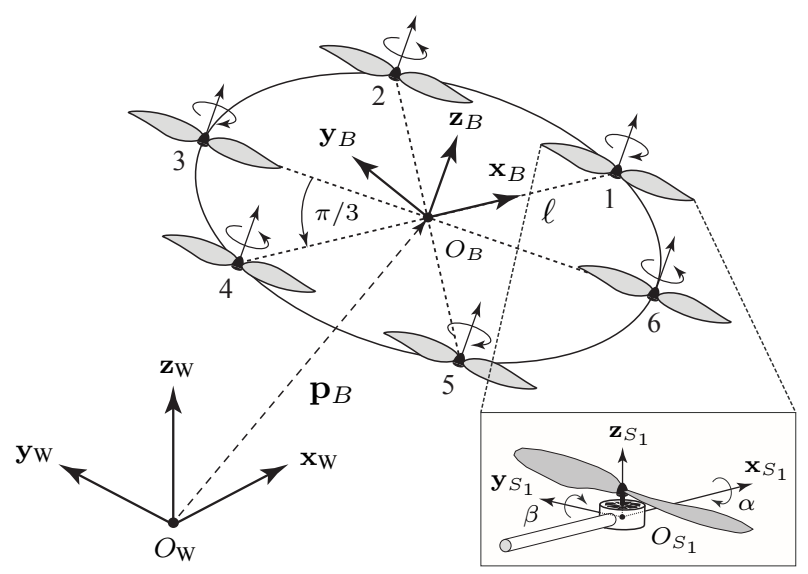

Fig. 1. Schematic representation of the hexarotor with dual-tilting propellers. The odd-numbered propellers spin clockwise and the even-numbered propellers spin counterclockwise. The inset shows the tilting angles $\alpha$ and $\beta$ of propeller 1 . 


$\mathbf{F}(\alpha, \beta)=\left[\begin{array}{cccccc}s \beta & \frac{1}{2} s \beta-\frac{\sqrt{3}}{2} c \beta s \alpha & -\frac{1}{2} s \beta+\frac{\sqrt{3}}{2} c \beta s \alpha & -s \beta & -\frac{1}{2} s \beta-\frac{\sqrt{3}}{2} c \beta s \alpha & \frac{1}{2} s \beta+\frac{\sqrt{3}}{2} c \beta s \alpha \\ -c \beta s \alpha & \frac{\sqrt{3}}{2} s \beta+\frac{1}{2} c \beta s \alpha & \frac{\sqrt{3}}{2} s \beta+\frac{1}{2} c \beta s \alpha & -c \beta s \alpha & -\frac{\sqrt{3}}{2} s \beta+\frac{1}{2} c \beta s \alpha & -\frac{\sqrt{3}}{2} s \beta+\frac{1}{2} c \beta s \alpha \\ c \beta c \alpha & c \beta c \alpha & c \beta c \alpha & c \beta c \alpha & c \beta c \alpha & c \beta c \alpha \\ c_{f}^{\tau} s \beta & -\frac{1}{2} c_{f}^{\tau} s \beta+\frac{\sqrt{3}}{2} V c \beta & -\frac{1}{2} c_{f}^{\tau} s \beta+\frac{\sqrt{3}}{2} V c \beta & c_{f}^{\tau} s \beta & -\frac{1}{2} c_{f}^{\tau} s \beta-\frac{\sqrt{3}}{2} V c \beta & -\frac{1}{2} c_{f}^{\tau} s \beta-\frac{\sqrt{3}}{2} V c \beta \\ -V c \beta & -\frac{\sqrt{3}}{2} c_{f}^{\tau} s \beta-\frac{1}{2} V c \beta & \frac{\sqrt{3}}{2} c_{f}^{\tau} s \beta+\frac{1}{2} V c \beta & V c \beta & -\frac{\sqrt{3}}{2} c_{f}^{\tau} s \beta+\frac{1}{2} V c \beta & \frac{\sqrt{3}}{2} c_{f}^{\tau} s \beta-\frac{1}{2} V c \beta \\ -W c \beta & W c \beta & -W c \beta & W c \beta & -W c \beta & W c \beta\end{array}\right]$

$$
V=\ell \cos \alpha+c_{f}^{\tau} \sin \alpha, \quad W=\ell \sin \alpha-c_{f}^{\tau} \cos \alpha,
$$

and $s \beta$ and $c \beta$ are shorthands for $\sin \beta$ and $\cos \beta$, respectively. Note that $V$ is the scalar projection of vector $\left[\ell, c_{f}^{\tau}\right]^{T}$ onto $[\cos \alpha, \sin \alpha]^{T}$ (i.e. onto the $\mathbf{y}_{S_{i}}$-axis), and that $W$ is the scalar projection of $\left[\ell, c_{f}^{\tau}\right]^{T}$ onto the orthogonal $[\sin \alpha,-\cos \alpha]^{T}$ (i.e. onto the $\mathbf{z}_{S_{i}}$-axis). We have that,

$$
\begin{aligned}
& \operatorname{det}(\mathbf{F}(\alpha, \beta))= \\
& -54 \cos \alpha \cos ^{2} \beta\left(V \sin \alpha \cos ^{2} \beta+c_{f}^{\tau} \sin ^{2} \beta\right)^{2} W,
\end{aligned}
$$

from which the singularities of $\mathbf{F}(\alpha, \beta)$ can be easily identified. In fact, $\operatorname{det}(\mathbf{F}(\alpha, \beta))=0$ if:

- $\underline{\text { Case 1: }} \cos \alpha=0$, i.e. $\alpha=(2 k+1) \pi / 2, k \in \mathbb{Z}$. In this case, $\operatorname{rank}(\mathbf{F}(\alpha, \beta))=5$ and its underactuation degree $^{2} \delta_{A}=1$. The spinning axes $\mathbf{z}_{S_{1}}, \ldots, \mathbf{z}_{S_{6}}$ of the six motors are coplanar. If $\beta=0$, they are tangent to the circular airframe (see Fig. 2(a), where $\alpha=\pi / 2$ ): if $f_{1}=f_{2}=\ldots=f_{6}$, the hexarotor stands still.

- Case 2: $\cos \beta=0$, i.e. $\beta=(2 k+1) \pi / 2, k \in \mathbb{Z}$. In this case, $\operatorname{rank}(\mathbf{F}(\alpha, \beta))=4$ and $\delta_{A}=2$ (see Fig. 2(b), where $\alpha=0$ and $\beta=-\pi / 2$ ). If $f_{1}=f_{2}=\ldots=f_{6}$, the hexarotor stands still.

- Case 3:

$$
V \sin \alpha \cos ^{2} \beta+c_{f}^{\tau} \sin ^{2} \beta=0 .
$$

Eq. (7) is transcendental and its zeros cannot be determined in closed form. However, for a fixed $\alpha \in$ $\left[k \pi-\arctan \left(\ell / c_{f}^{\tau}\right), k \pi\right], \quad k \in \mathbb{Z}$, we have $\beta=$ $\pm \arctan \left(\sqrt{-\frac{V}{c_{f}^{\tau}} \sin \alpha}\right)$. For these pairs of $\alpha$ and $\beta$, $\operatorname{rank}(\mathbf{F}(\alpha, \beta))=4$ and $\delta_{A}=2$. Two values of $\alpha$ are of special interest:

- $\underline{\text { Case 3.1: }} V=0$, i.e. $\alpha=-\arctan \left(\ell / c_{f}^{\tau}\right)$ (cf. [20, Sect. IIIB]). Note that $\ell / c_{f}^{\tau}>0$, therefore $\arctan \left(\ell / c_{f}^{\tau}\right)>0$.

- Case 3.2: $\sin \alpha=0$, i.e. $\alpha=k \pi, k \in \mathbb{Z}$. If $\alpha=0$, the spinning axes $\mathbf{z}_{S_{1}}, \ldots, \mathbf{z}_{S_{6}}$ of the six motors are parallel to $\mathbf{z}_{B}$ and the platform behaves as a conventional hexarotor.

- Case 4: $W=0$, i.e.

$$
\alpha=\arctan \left(c_{f}^{\tau} / \ell\right) .
$$

In this case, $\operatorname{rank}(\mathbf{F}(\alpha, \beta))=5$ and $\delta_{A}=1$.

\footnotetext{
${ }^{2}$ The underactuation degree $\delta_{A}$ of a mechanical system modeled as $\ddot{\mathbf{q}}=$ $\boldsymbol{f}(\mathbf{q}, \dot{\mathbf{q}}, \boldsymbol{u})$, where $\mathbf{q}$ is the vector of generalized coordinates and $\boldsymbol{u}$ is the input vector, is the difference between the dimension of $\mathbf{q}$ and the rank of the Jacobian matrix $\partial \boldsymbol{f} / \partial \boldsymbol{u}$, which, in the case under examination, is equal to the rank of $\mathbf{F}$. If $\delta_{A}=0$, the system is said fully actuated.
}

Remark 1 (Force and torque underactuation): Note that the singularities in Case 1 and Case 3.2 are sources of force underactuation for the hexarotor, whereas those in Case 3.1 and Case 4 of torque underactuation. On the other hand, the singularities in Case 2 cause both force and torque underactuation (cf. the 3rd and 6th row of $\mathbf{F}(\alpha, \beta)$ ).

Remark 2: With reference to eq. (6), for $\beta=0$, $\operatorname{det}(\mathbf{F}(\alpha, 0))=-54 V^{2} W \cos \alpha \sin ^{2} \alpha$ (cf. Fig. 3), while for $\alpha=0, \operatorname{det}(\mathbf{F}(0, \beta))=54\left(c_{f}^{\tau}\right)^{3} \cos ^{2} \beta \sin ^{4} \beta$.

\section{Geometric interpretation of torque singularities}

Note that while the singularities of Case 1 and 2 can be easily avoided, since they correspond to orientations of the propellers of scarce practical interest, the singularities of Case 3.1 and Case 4 are subtler. In what follows, we will only focus on Case 4, since Case 3.1 is complementary. Fig. 2(c) reports the forces and torques generated by propeller $i$ which is tilted of an angle $\alpha_{i}=(-1)^{i-1} \alpha>0$ about the $\mathbf{x}_{S_{i}}$-axis, $i \in\{1, \ldots, 6\}$ (the axis is directed out of the page at the reader). When propeller $i$ rotates about its spinning axis $\mathbf{z}_{S_{i}}$, it generates a thrust force $\mathbf{f}_{i}$ which, in turn, produces a torque $\tau_{f_{i}}$ at the CoM of the platform and a drag torque $\tau_{d_{i}}$. The magnitude of these vectors can be expressed as [21]:

$$
\begin{aligned}
f_{i} & =c_{f} w_{i}^{2}, \\
\tau_{f_{i}} & =\ell f_{i}=\ell c_{f} w_{i}^{2}, \\
\tau_{d_{i}} & =c_{\tau} w_{i}^{2},
\end{aligned}
$$

where $w_{i}$ is the angular velocity of propeller $i$ about the $\mathbf{z}_{S_{i}}$-axis, and $c_{f}$ and $c_{\tau}$ are the estimated thrust and aerodynamic drag factors of the propeller, respectively. Note that (9a)-(9c) is an approximation of a more accurate aerodynamic model, which is, however, appropriate for the range of $w_{i}$ 's of interest in this paper (between 0 and $750 \mathrm{rad} / \mathrm{s}$, cf. Sect. IV). To gain some physical insight into condition (8), it is convenient to study the total torque $\boldsymbol{\tau}_{i}$ applied at the CoM of the hexarotor by propeller $i$, which is given by $\boldsymbol{\tau}_{i}=\boldsymbol{\tau}_{f_{i}}+\boldsymbol{\tau}_{d_{i}}$, where the two torque components are always orthogonal, by construction. From Fig. 2(c) (see the yellow-shaded rectangle), we deduce that:

$$
\begin{aligned}
\gamma_{i}+\mu_{i} & =\pi / 2, \\
\tan \gamma_{i} & =\tau_{f_{i}} / \tau_{d_{i}} .
\end{aligned}
$$

By plugging eqs.(10) into (11), we find that $\tan \mu_{i}=$ $\tau_{d_{i}} / \tau_{f_{i}}$. Setting $c_{f}^{\tau}=c_{\tau} / c_{f}$, substitution of (9b)-(9c) into the previous equation yields $\tan \mu_{i}=c_{f}^{\tau} / \ell$, which tells us 


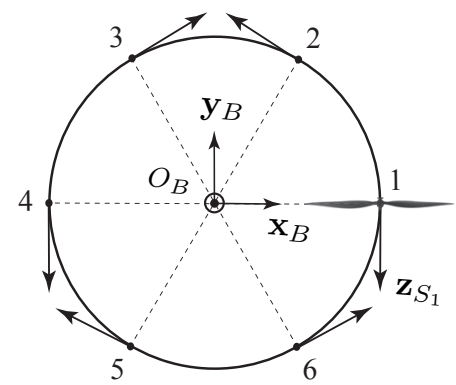

(a)

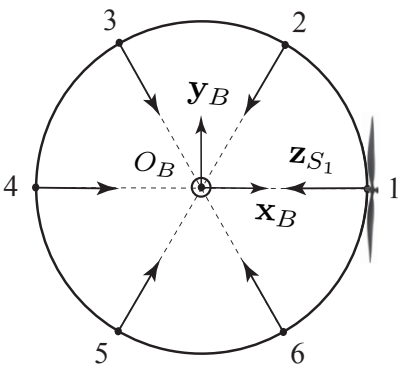

(b)

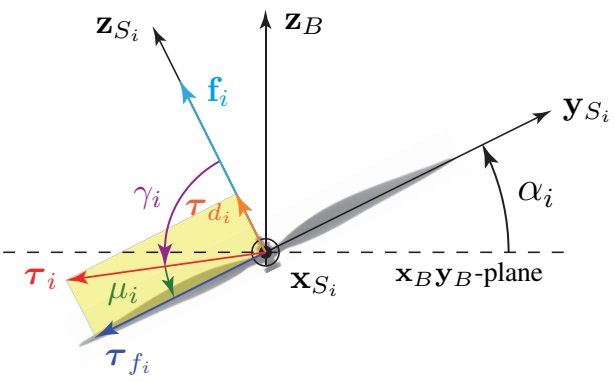

(c)

Fig. 2. Three of the four singular configurations of the hexarotor with dual-tilting propellers: (a) Case 1 for $\alpha=\pi / 2$ and $\beta=0$ (top view); (b) Case 2 for $\alpha=0$ and $\beta=-\pi / 2$ (top view); (c) Case 4: illustration of the forces and torques generated by propeller $i$. The propeller spins clockwise $\left(w_{i}<0\right.$ ) with a tilting angle $\alpha_{i}>0$ about the $\mathbf{x}_{S_{i}}$-axis. The $\mathbf{x}_{B} \mathbf{y}_{B}$-plane is dashed.

that the direction of the total torque generated by propeller $i$ at the CoM of the hexarotor only depends on two physical parameters, $c_{f}^{\tau}$ and $\ell$, and on the tilting angle $\alpha$. Notably, the direction of $\boldsymbol{\tau}_{i}$ does not depend on the angular velocity of the propeller itself. If $\mu_{i}=\alpha_{i}$, then $\boldsymbol{\tau}_{i}$ belongs to the $\mathbf{x}_{B} \mathbf{y}_{B}$-plane and propeller $i$ cannot generate torques along $\mathbf{z}_{B}$ anymore (see Fig. 2(c)). If this condition holds for all propellers, i.e. $\mu_{i}=\left|\alpha_{i}\right|, \forall i$, then the entries of the 6th row of $\mathbf{F}(\alpha, \beta)$ (which maps $\boldsymbol{u}$ onto torques about the $\mathbf{z}_{B^{-}}$ axis) are zero and the matrix becomes rank-one deficient. This corresponds to condition (8), previously obtained in a purely algebraic fashion. Note that Case 3.1 is even more critical than Case 4 , since this time $\tau_{i}$ is aligned with $\mathbf{z}_{B}$, yielding a rank-two deficient $\mathbf{F}(\alpha, \beta)$ (the 4 th and 5th row become zero). Fig. 4 complements Fig. 2(c) by reporting the other possible combinations of forces and torques that propeller $i$ can generate according to the signs of $\alpha_{i}$ and $w_{i}$.

\section{Tilt-planner design}

Note that since $\alpha$ and $\beta$ are slowly-varying parameters, their value can be decided by a high-level tilt planner or by a human operator. The angle $\alpha$ can be gradually changed during flight: as a result, a continuum of configurations, ranging from $\delta_{A}=2$ to $\delta_{A}=0$, is spanned (cf. Fig. 3). The hexarotor can thus adapt its morphology to respond to variable external conditions or task-related demands. In particular, in a fully-actuated platform position and attitude can be independently controlled and since any wrench can be counteracted during contact with the environment, aerial physical interaction becomes possible [18]. Note that $c_{f}^{\tau}=c_{\tau} / c_{f}$ is "small", since the aerodynamic drag factor $c_{\tau}$ is typically one order of magnitude smaller than $c_{f}$.

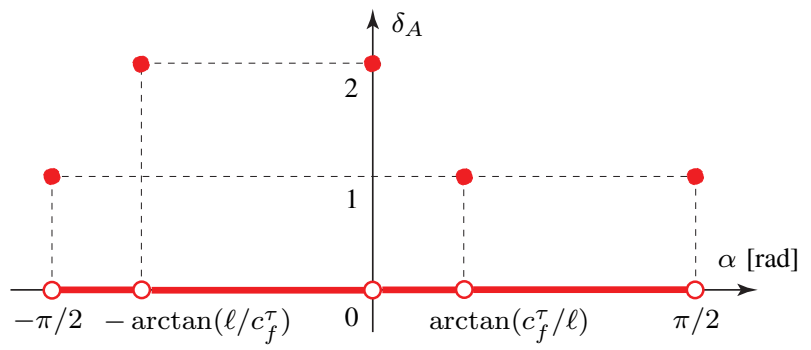

Fig. 3. Values assumed by $\delta_{A}$ for $\alpha \in[-\pi / 2, \pi / 2]$ and $\beta=0$.
As a consequence, $\alpha=\arctan \left(c_{f}^{\tau} / \ell\right)$ is "small" as well. For instance, if we take $c_{f}^{\tau}=1.7 \times 10^{-2} \mathrm{~m}$ and $\ell=0.315 \mathrm{~m}$ as in [14], we obtain a critical $\alpha=3.0892^{\circ}$. Hence, if one decides to increase $\alpha$ starting from zero in order to transform the hexarotor into a fully-actuated platform, a singularity will be crossed. This results in a temporary loss of maneuverability, but the hexarotor will be able to maintain stable flight long enough for the tilt planner to escape from the narrow zone of torque underactuation. Finally, note that since the implementation of the tilt planner is necessarily task-specific, its design and that of the optimal controller (see Sect. III-B) will be decoupled, and we will assume that a singularity-free, smooth profile for $\alpha(t)$ and $\beta(t)$ has been precomputed to comply with the task requirements.

\section{DETERMINATION OF MINIMUM-ENERGY TRAJECTORIES}

\section{A. Electrical model of a BLDC motor}

The model for a BLDC motor of the hexarotor takes into account the energy dissipated in the resistive and inductive windings, and the energy required to overcome the internal and load friction [13]. The instantaneous current $i(t)$ in the motor is given by (see Fig. 5),

$\mathrm{i}(t)=\frac{1}{K_{\mathrm{T}}}\left[T_{f}+T_{\mathrm{L}}(w(t))+D_{f} w(t)+\left(J_{\mathrm{m}}+J_{\mathrm{L}}\right) \frac{d w(t)}{d t}\right]$

where $w(t)$ is the angular velocity of the motor shaft $[\mathrm{rad} / \mathrm{s}]$, $K_{\mathrm{T}}$ is the torque constant of the motor $[\mathrm{Nm} / \mathrm{A}], T_{f}$ is the motor friction torque, $T_{\mathrm{L}}(w(t))$ is the speed-dependent load friction torque which results from propeller drag, $D_{f}$ is the viscous damping coefficient of the motor [ $\mathrm{Nms} / \mathrm{rad}]$, and $J_{\mathrm{m}}$ and $J_{\mathrm{L}}$ are the motor and load moments of inertia, respectively. The voltage $\mathrm{e}(t)$ across the motor is given by:

$$
\mathrm{e}(t)=R \mathrm{i}(t)+K_{\mathrm{E}} w(t)+L \frac{d \mathrm{i}(t)}{d t},
$$

where $R$ and $L$ are the resistance and inductance of phase winding, respectively, and $K_{\mathrm{E}}$ is the voltage constant of the motor [Vs/rad]. Note that $K_{\mathrm{E}}=K_{\mathrm{T}}$ [13]: in addition, if $K_{\mathrm{E}}$ is expressed in $\mathrm{mV} / \mathrm{rpm}$, we have that $K_{\mathrm{E}}=1000 / K_{\mathrm{V}}$ where $K_{\mathrm{V}}$ is the motor velocity constant $[\mathrm{rpm} / \mathrm{V}]$. Since the resistance $R_{L}$, representing the losses in the magnetic circuit of the motor, is usually much larger than $R$, the effect of $R_{L}$ on motor operation can be neglected. Under steady-state 


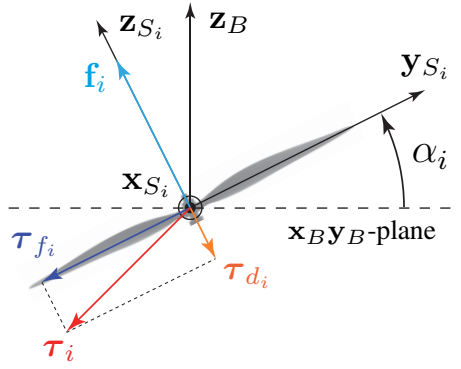

(a)

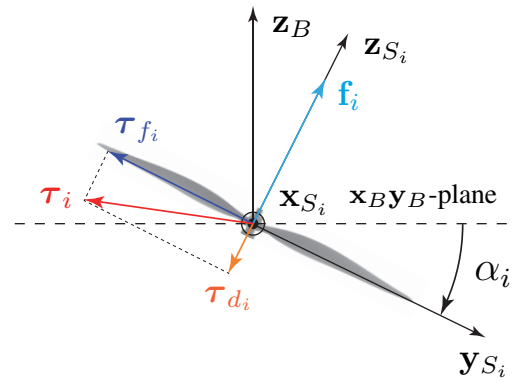

(b)

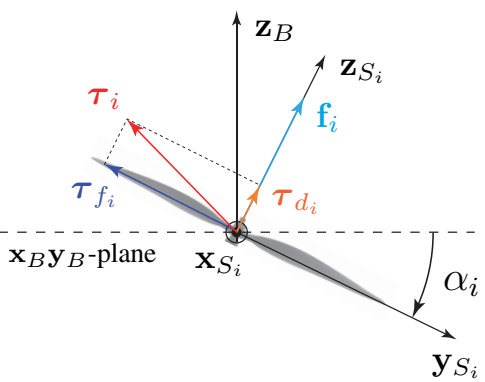

(c)

Fig. 4. Case 4: illustration of the other possible combinations of forces and torques that propeller $i$ can generate (cf. Fig. $2(\mathrm{c})$ ). (a) $\alpha_{i}>0$ and $w_{i}>0$; (b) $\alpha_{i}<0$ and $w_{i}>0$; (c) $\alpha_{i}<0$ and $w_{i}<0$. The $\mathbf{x}_{B} \mathbf{y}_{B}$-plane is dashed.

conditions, the current $i(t)$ is constant and (13) reduces to:

$$
\mathrm{e}(t)=R \mathrm{i}(t)+K_{\mathrm{E}} w(t)
$$

where $\mathrm{e}_{g}(t)=K_{\mathrm{E}} w(t)$ is the counter electromotive force of the motor.

\section{B. Optimal control problem}

We can now introduce an optimal control problem for system (1), whose solution will give us the minimumenergy trajectory of the hexarotor between a given initial and final configuration. Let $\mathrm{e}_{i}(t)$ and $\boldsymbol{i}_{i}(t)$ denote the voltage and the current across motor $i \in\{1, \ldots, 6\}$ at time $t$. The energy consumed by the hexarotor between the initial time $t_{0}$ and the fixed end time $t_{\mathrm{f}}$ is then,

$$
\mathcal{E}=\int_{t_{0}}^{t_{\mathrm{f}}} \sum_{i=1}^{6} \mathrm{e}_{i}(t) \dot{i}_{i}(t) d t
$$

By leveraging (12) and (14) for the six identical motors, and by noticing that $T_{\mathrm{L}}\left(w_{i}(t)\right)=\tau_{d_{i}}(t), i \in\{1, \ldots, 6\}$ (recall (9c)), we can rewrite (15) as follows:

$$
\mathcal{E}=\int_{t_{0}}^{t_{\mathrm{f}}} \sum_{i=1}^{6}\left[b_{1}+b_{2} w_{i}(t)+b_{3} w_{i}^{2}(t)+b_{4} w_{i}^{3}(t)+b_{5} w_{i}^{4}(t)\right.
$$$$
\left.+b_{6} \dot{w}_{i}(t)+b_{7} \dot{w}_{i}^{2}(t)+b_{8} w_{i}(t) \dot{w}_{i}(t)+b_{9} w_{i}^{2}(t) \dot{w}_{i}(t)\right] d t
$$

where the positive constants $b_{1}, \ldots, b_{9}$ are given by:

$$
\begin{aligned}
b_{1} & =\frac{R T_{f}^{2}}{K_{\mathrm{T}}^{2}}, \quad b_{2}=\frac{T_{f}}{K_{\mathrm{T}}}\left(\frac{2 R D_{f}}{K_{\mathrm{T}}}+K_{\mathrm{E}}\right), \\
b_{3} & =\frac{D_{f}}{K_{\mathrm{T}}}\left(\frac{R D_{f}}{K_{\mathrm{T}}}+K_{\mathrm{E}}\right)+\frac{2 R T_{f} c_{\tau}}{K_{\mathrm{T}}^{2}}, \\
b_{4} & =\frac{c_{\tau}}{K_{\mathrm{T}}}\left(\frac{2 R D_{f}}{K_{\mathrm{T}}}+K_{\mathrm{E}}\right), \quad b_{5}=\frac{R c_{\tau}^{2}}{K_{\mathrm{T}}^{2}}, \quad b_{6}=\frac{2 R J_{t} T_{f}}{K_{\mathrm{T}}^{2}}, \\
b_{7} & =\frac{R J_{t}^{2}}{K_{\mathrm{T}}^{2}}, \quad b_{8}=\frac{J_{t}}{K_{\mathrm{T}}}\left(\frac{2 R D_{f}}{K_{\mathrm{T}}}+K_{\mathrm{E}}\right), \quad b_{9}=\frac{2 R J_{t} c_{\tau}}{K_{\mathrm{T}}^{2}},
\end{aligned}
$$

being $J_{t}=J_{\mathrm{m}}+J_{\mathrm{L}}$ the total inertia of a motor.

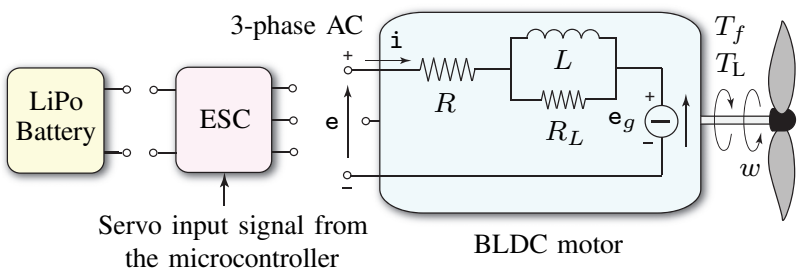

Fig. 5. Electrical model of a BLDC motor of the hexarotor.
Remark 3 (Simplified cost function): Note that,

$$
\begin{gathered}
\int_{t_{0}}^{t_{\mathrm{f}}} \sum_{i=1}^{6}\left[b_{6} \dot{w}_{i}(t)+b_{8} w_{i}(t) \dot{w}_{i}(t)+b_{9} w_{i}^{2}(t) \dot{w}_{i}(t)\right] d t \\
=\sum_{i=1}^{6}\left[b_{6}\left(w_{i}\left(t_{\mathrm{f}}\right)-w_{i}\left(t_{0}\right)\right)+\frac{1}{2} b_{8}\left(w_{i}^{2}\left(t_{\mathrm{f}}\right)-w_{i}^{2}\left(t_{0}\right)\right)\right. \\
\left.\quad+\frac{1}{3} b_{9}\left(w_{i}^{3}\left(t_{\mathrm{f}}\right)-w_{i}^{3}\left(t_{0}\right)\right)\right] .
\end{gathered}
$$

Therefore, if we assume that $w_{i}\left(t_{0}\right)=w_{i}\left(t_{\mathrm{f}}\right), \forall i$, i.e. the initial and final angular velocity of each motor are identical, eq. (16) reduces to:

$\varepsilon_{r}=\int_{t_{0}}^{t_{\mathrm{f}}} \sum_{i=1}^{6}\left[b_{1}+b_{2} w_{i}+b_{3} w_{i}^{2}+b_{4} w_{i}^{3}+b_{5} w_{i}^{4}+b_{7} \dot{w}_{i}^{2}\right] d t$,

where the time index $t$ has been dropped. This simplified cost function will be used in the rest of this section.

We now cast the minimum-energy trajectory generation problem as a standard optimal control problem [22]. To this end, we rewrite system (1) in state-space form by introducing the state vector $\mathbf{x}=\left[x_{1}, \ldots, x_{18}\right]^{T} \in \mathbb{R}^{18}$ and the auxiliary control input $\boldsymbol{\eta}=\left[\eta_{1}, \ldots, \eta_{6}\right]^{T} \in \mathbb{R}^{6}$, via dynamic extension,

$x_{1}=x, x_{2}=\dot{x}_{1}, x_{3}=y, x_{4}=\dot{x}_{3}, x_{5}=z, x_{6}=\dot{x}_{5}$

$x_{7}=\phi, x_{8}=\dot{x}_{7}, x_{9}=\theta, x_{10}=\dot{x}_{9}, x_{11}=\psi, x_{12}=\dot{x}_{11}$,

$x_{13}=w_{1}, \dot{x}_{13}=\eta_{1}, \ldots, x_{18}=w_{6}, \dot{x}_{18}=\eta_{6}$,

where $\phi, \theta$ and $\psi$ are the hexarotor's Euler (roll, pitch and yaw) angles. This yields the following nonlinear system affine in the auxiliary control input $\boldsymbol{\eta}$ :

$\left\{\begin{array}{l}\dot{x}_{1}=x_{2}, \dot{x}_{3}=x_{4}, \dot{x}_{5}=x_{6}, \\ {\left[\begin{array}{c}\dot{x}_{2} \\ \dot{x}_{4} \\ \dot{x}_{6}\end{array}\right]=-g \boldsymbol{e}_{3}+\frac{c_{f}}{m} \mathbf{R}_{B}\left(x_{7}, x_{9}, x_{11}\right) \mathbf{F}_{1}(\alpha, \beta)\left[\begin{array}{c}x_{13}^{2} \\ \vdots \\ x_{18}^{2}\end{array}\right],} \\ \dot{x}_{7}=x_{8}, \dot{x}_{9}=x_{10}, \dot{x}_{11}=x_{12}, \\ {\left[\begin{array}{c}\dot{x}_{8} \\ \dot{x}_{10} \\ \dot{x}_{12}\end{array}\right]=\mathrm{J}^{-1}\left(-\left[\begin{array}{c}x_{8} \\ x_{10} \\ x_{12}\end{array}\right] \times \mathrm{J}\left[\begin{array}{c}x_{8} \\ x_{10} \\ x_{12}\end{array}\right]+c_{f} \mathbf{F}_{2}(\alpha, \beta)\left[\begin{array}{c}x_{13}^{2} \\ \vdots \\ \dot{x}_{13}=\eta_{1}, \ldots, \dot{x}_{18}=\eta_{6},\end{array}\right]\right),}\end{array}\right.$

where eq. (9a) has been used and $\mathbf{R}_{B}\left(x_{7}, x_{9}, x_{11}\right)=$ $\mathbf{R}_{z}\left(x_{11}\right) \mathbf{R}_{y}\left(x_{9}\right) \mathbf{R}_{x}\left(x_{7}\right)$.

Assumption 1: The singularity-free, smooth profile of $\alpha(t), \beta(t), t \in\left[t_{0}, t_{\mathrm{f}}\right]$, has been computed by the tilt planner. 
The profile complies with the task requirements and it is known at the time of solving problem (19) below.

Under Assumption 1, we can finally introduce the following optimal control problem by combining (17) with system (18):

$$
\begin{array}{ll}
\min _{\boldsymbol{\eta}} & \mathcal{E}_{r}=\int_{t_{0}}^{t_{\mathrm{f}}}\left[\sum_{k=13}^{18}\left(b_{1}+b_{2} x_{k}+b_{3} x_{k}^{2}+b_{4} x_{k}^{3}+b_{5} x_{k}^{4}\right)\right. \\
& \left.+b_{7} \boldsymbol{\eta}^{T} \boldsymbol{\eta}\right] d t
\end{array}
$$

where $w_{\max }>0$ is the velocity saturation of the motors and $\mathbf{x}_{t_{0}}, \mathbf{x}_{t_{\mathrm{f}}} \in \mathbb{R}^{18}$ are assigned boundary states. Note that the last six components of $\mathbf{x}_{t_{0}}$ and $\mathbf{x}_{t_{\mathrm{f}}}$ must match in order to fulfill the condition of Remark 3 .

\section{NUMERICAL EXPERIMENTS}

To validate our optimal control scheme, we considered the physical parameters of the FAST-Hex hexarotor [14] (see Table I). The FAST-Hex is equipped with six MikroKopter MK3638 brushless outrunner motors. We computed the inertia $J_{\mathrm{m}}$ of the motors using the inertia formula of a thin cylindrical shell with open ends of radius $r_{\text {rot }}$ and mass $m_{\text {rot }}$ (i.e. $J_{\mathrm{m}}=m_{\text {rot }} r_{\text {rot }}^{2}$ ), assuming that the weight of the rotating part of the motor is $50 \%$ of the total weight. Following [21], we know that $J_{\mathrm{L}}=\frac{1}{4} n_{\mathrm{bl}} m_{\mathrm{bl}}(r-\epsilon)^{2}$ where $n_{\mathrm{bl}}$ is the number of blades of the propeller, $m_{\mathrm{bl}}$ is the blade mass, $r$ is the radius of the propeller, and $\epsilon$ is the offset between the blade root and the motor hub. The total mass of the FAST-Hex, $m=2.4 \mathrm{~kg}$, includes the mass of the $4 \mathrm{~s}$ LiPo $14.8 \mathrm{~V}, 2200 \mathrm{mAh}$ battery $(0.238 \mathrm{~kg})$ and of the six propeller units $(6 \times 0.15 \mathrm{~kg}=0.9 \mathrm{~kg})$. A diagonal inertia matrix $\mathrm{J}=\operatorname{diag}\left(\mathrm{J}_{x}, \mathrm{~J}_{y}, \mathrm{~J}_{z}\right)$ was considered for the platform. Problem (19) has been numerically solved using the TOMLAB Optimization Environment under Matlab 7.9. TOMLAB/PROPT relies on pseudospectral (Gauss or Chebyshev) collocation methods for solving complex optimal control problems [19]. In our first test, we solved problem (19) to find the minimum-energy control input $\boldsymbol{\eta}(t)$ to fly from the origin at time $t_{0}=0 \mathrm{~s}$ to the point of coordinates $[4,6,8]^{T} \mathrm{~m}$ with a yaw angle of $45^{\circ}$, at the fixed end time $t_{\mathrm{f}}=15 \mathrm{~s}$. This corresponds to $\mathbf{x}_{t_{0}}=\left[\mathbf{0}_{1 \times 12}, \omega_{\mathrm{h}}, \ldots, \omega_{\mathrm{h}}\right]^{T}$ and $\mathbf{x}_{t_{\mathrm{f}}}=\left[4,0,6,0,8, \mathbf{0}_{1 \times 5}, \pi / 4,0, \omega_{\mathrm{h}}, \ldots, \omega_{\mathrm{h}}\right]^{T}$ where $\omega_{\mathrm{h}}=356.047 \mathrm{rad} / \mathrm{s} \simeq 3400 \mathrm{rpm}$ (note that with $\alpha=\beta=0^{\circ}$, the required velocity of a propeller for static hover is

\begin{tabular}{|l|l|}
\hline$K_{\mathrm{E}}=9.5493 / K_{\mathrm{V}} \mathrm{Vs} / \mathrm{rad}$ & $K_{\mathrm{V}}=760 \mathrm{rpm} / \mathrm{V}, R=0.07 \Omega$ \\
\hline$T_{f}=4 \times 10^{-2} \mathrm{Nm}$ & $D_{f}=2 \times 10^{-4} \mathrm{Nms} / \mathrm{rad}$ \\
\hline$r_{\mathrm{rot}}=0.0175 \mathrm{~m}, m_{\mathrm{rot}}=0.05 \mathrm{~kg}$ & $J_{\mathrm{m}}=1.531 \times 10^{-5} \mathrm{kgm}^{2}$ \\
\hline$c_{f}=9.902 \times 10^{-4} \mathrm{Ns}^{2}$ & $n_{\mathrm{bl}}=2, m_{\mathrm{bl}}=0.007 \mathrm{~kg}$ \\
\hline$c_{f}^{\tau}=1.91 \times 10^{-2} \mathrm{~m}$ & $c_{\tau}=1.896 \times 10^{-5} \mathrm{Nms}^{2}$ \\
\hline$r=0.1524 \mathrm{~m}, \epsilon=0.004 \mathrm{~m}$ & $w_{\max }=750 \mathrm{rad} / \mathrm{s}$ \\
\hline$\ell=0.315 \mathrm{~m}, m=2.4 \mathrm{~kg}$ & $\mathrm{~J}_{x}=1.1549 \times 10^{-2} \mathrm{~kg} \mathrm{~m}^{2}$ \\
\hline $\mathrm{J}_{y}=1.1368 \times 10^{-2} \mathrm{kgm}^{2}$ & $\mathrm{~J}_{z}=1.9444 \times 10^{-2} \mathrm{kgm}^{2}$ \\
\hline
\end{tabular}

TABLE I

Parameters of the FAST-Hex platform [14]. $\left.2 \pi \sqrt{\frac{m g}{6 c_{f}}}=395.483 \mathrm{rad} / \mathrm{s}\right)$. With these boundary states, the condition of Remark 3 is satisfied. Fig. 6(a) shows the minimum-energy trajectory of the FAST-Hex for $\alpha(t)=$ $\beta(t)=0^{\circ}$ (solid black) and $\alpha(t)=55^{\circ}, \beta(t)=15^{\circ}$ (dashed black), $t \in\left[t_{0}, t_{\mathrm{f}}\right]$. To obtain accurate trajectories, problem (19) was first solved on a sparse grid, and then re-solved recursively by using the obtained solution as a starting point. The successively finer grids correspond to a larger number of collocation points: 20,40 and 60 . The starting guess was $\mathbf{x}\left(t_{0}\right)=\mathbf{x}_{t_{0}}, \boldsymbol{\eta}\left(t_{0}\right)=\mathbf{0}_{6 \times 1}$. Figs. 6(b)-(c) report the time evolution of the position and velocity of the FAST-Hex for $\alpha(t)=\beta(t)=0^{\circ}$ (solid) and $\alpha(t)=55^{\circ}$, $\beta(t)=15^{\circ}$ (dashed), respectively. In both cases, PROPT invoked the nonlinear solver SNOPT and the optimality conditions were satisfied: in the first (second) case, the cost function was evaluated 806 (645) times, and the total energy consumed was $1.6933 \mathrm{~kJ}(2.6404 \mathrm{~kJ})$. Fig. 6(a) also reports the trajectory of the FAST-Hex for $\alpha(t)=55^{\circ}, \beta(t)=15^{\circ}$ (green), generated with the nonlinear controller considered in [13, Sect. IVA], that we tailored to system (18). Using the same control gains as in [13], we obtained a consumption of $3.0745 \mathrm{~kJ}$ which corresponds to a $16.44 \%$ increase with respect to the energy-optimal trajectory.

Finally, to evaluate the impact of $\alpha$ on energy consumption, we considered the same boundary conditions and parameters as above, and we computed the minimum $\mathcal{E}_{r}$ for $\alpha \in\left(-90^{\circ}, 90^{\circ}\right)$ by considering a step size of $1^{\circ}$ and setting $\beta=0^{\circ}$ (cf. Fig. 3). The number of collocation points was fixed to 40 , which offered a good trade-off between solution accuracy and computation overhead. From Figs. 6(d)-(e), we observe that the conventional hexarotor configuration $\left(\alpha=0^{\circ}\right)$ is the most energetically efficient $(1.6495 \mathrm{~kJ})$, that a $1^{\circ}$ variation in $\alpha$ yields, on average, a $70.5 \mathrm{~J}$ variation in the figure of merit, and that the consumption curve is near symmetrical about the origin. Although further work is necessary to show that for any possible pair of boundary states, the fully-actuated configuration is always less energy-efficient than the conventional one, our results suggest that higher maneuverability entails increased energy consumption. Note that the peak in Fig. 6(d) corresponds to $\alpha=-87^{\circ}$. For the contiguous critical $\alpha=$ $-(180 / \pi) \arctan \left(\ell / c_{f}^{\tau}\right)=-86.5215^{\circ}$ (recall Case 3.1 in Sect. II-B), the iteration limit was reached, and no feasible solution was found by the SNOPT solver. On the other hand, for $\alpha=(180 / \pi) \arctan \left(c_{f}^{\tau} / \ell\right)=3.4785^{\circ}$ (cf. Case 4$)$, the FAST-Hex consumed $1.7191 \mathrm{~kJ}$.

\section{CONCLUSIONS AND FUTURE WORK}

In this paper, we have considered a hexarotor with dualtilting propellers and we have determined minimum-energy trajectories between two given boundary states, by solving an optimal control problem which explicitly accounts for the electrical parameters of the six motors. We have also fully characterized the underactuation degree of the hexarotor by studying the rank deficiency of the control allocation matrix.

In future works, we plan to perform a comparative study with the minimum snap trajectory planner in [23], and to solve our optimal control problem on the FAST-Hex on-board computer. We are also interested in shedding light 


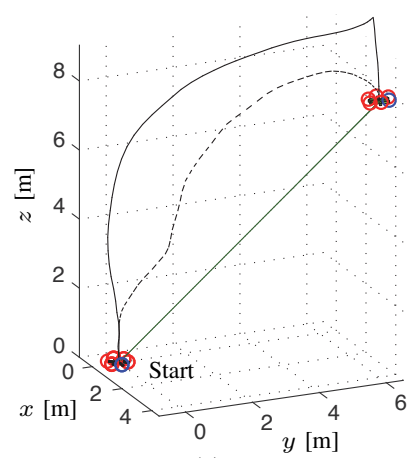

(a)

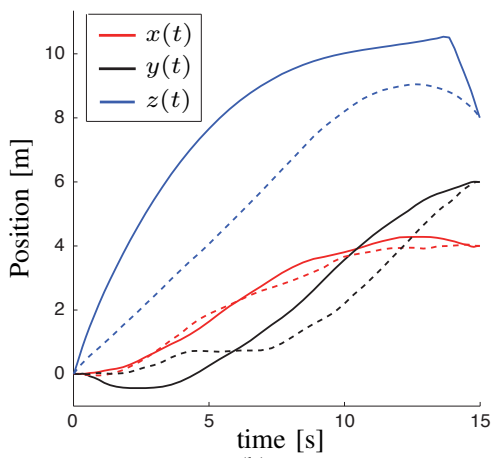

(b)

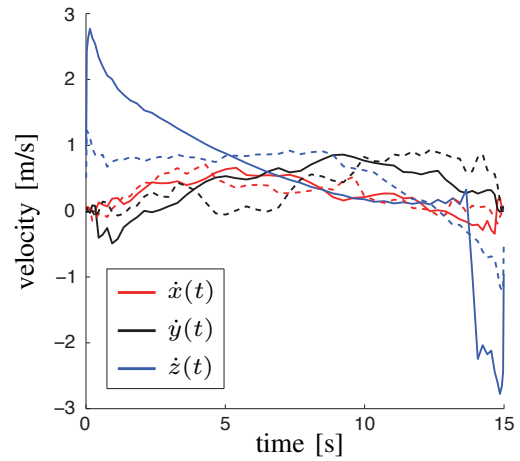

(c)

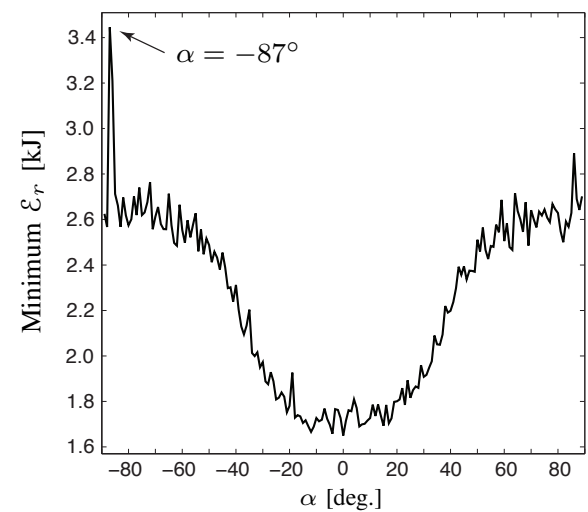

(d)

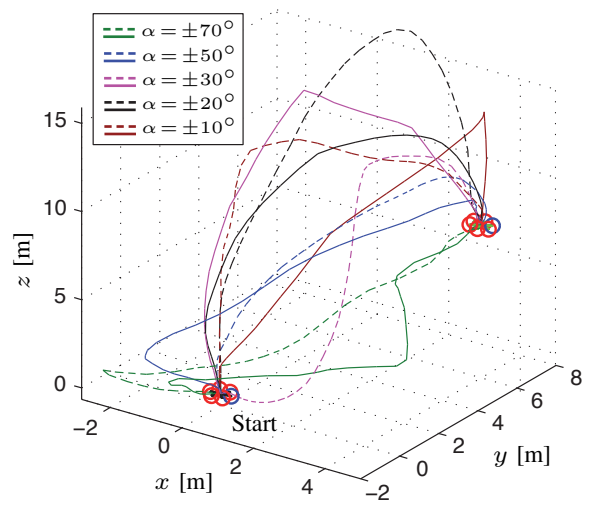

(e)

Fig. 6. (a) Minimum-energy trajectory of the FAST-Hex for $\alpha(t)=\beta(t)=0^{\circ}$ (solid black) and $\alpha(t)=55^{\circ}, \beta(t)=15^{\circ}$ (dashed black), $t \in\left[t_{0}, t_{\mathrm{f}}\right]$. The green trajectory is generated by the nonlinear regulator in [13, Sect. IVA] for $\alpha(t)=55^{\circ}, \beta(t)=15^{\circ}$ (propeller 1 is marked in blue); Time evolution of (b) the position, and (c) the velocity of the FAST-Hex for $\alpha(t)=\beta(t)=0^{\circ}$ (solid) and $\alpha(t)=55^{\circ}, \beta(t)=15^{\circ}$ (dashed) using our method. (d) Energy consumed by the FAST-Hex for $\alpha \in\left(-90^{\circ}, 90^{\circ}\right), \beta=0^{\circ}$, and (e) ten of the corresponding optimal trajectories.

on the "internal motions" of a statically hovering hexarotor with dual-tilting propellers: in fact, we would like to identify those profiles of $\alpha(t)$ and $\beta(t)$, treated as additional control inputs, which result in a zero net motion of the platform.

\section{REFERENCES}

[1] G. Avanzini, E.L. de Angelis, and F. Giulietti. Optimal performance and sizing of a battery-powered aircraft. Aerosp. Sci. Technol., 59:132144, 2016

[2] J. Thomas, G. Loianno, K. Daniilidis, and V. Kumar. Visual Servoing of Quadrotors for Perching by Hanging From Cylindrical Objects. IEEE Robot. Autom. Lett., 1(1):57-64, 2016.

[3] M.T. Pope et al. A Multimodal Robot for Perching and Climbing on Vertical Outdoor Surfaces. IEEE Trans. Robot., 33(1):38-48, 2017.

[4] S. Driessens and P. Pounds. The Triangular Quadrotor: A More Efficient Quadrotor Configuration. IEEE Trans. Robot., 31(6):15171526, 2015.

[5] T. Chang and H. Yu. Improving Electric Powered UAVs' Endurance by Incorporating Battery Dumping Concept. Procedia Engineering, 99:168-179, 2015.

[6] M.M. Nicotra, R. Naldi, and E. Garone. Nonlinear control of a tethered UAV: The taut cable case. Automatica, 78:174-184, 2017.

[7] A. Abdilla, A. Richards, and S. Burrow. Power and Endurance Modelling of Battery-Powered Rotorcraft. In Proc. IEEE/RSJ Int. Conf. Intel. Robots Syst., pages 675-680, 2015.

[8] M. Gatti, F. Giulietti, and M. Turci. Maximum endurance for batterypowered rotary-wing aircraft. Aerosp. Sci. Technol., 45:174-179, 2015.

[9] A.B. Junaid, Y. Lee, and Y. Kim. Design and implementation of autonomous wireless charging station for rotary-wing UAVs. Aerosp. Sci. Technol., 54:253-266, 2016.

[10] G.M. Plaizier, E. Andersen, B. Truong, X. He, S. Roundy, and K.K. Leang. Design, Modeling, and Analysis of Inductive Resonant Coupling Wireless Power Transfer for Micro Aerial Vehicles (MAVs). In Proc. IEEE Int. Conf. Robot. Automat., pages 6104-6109, 2018.
[11] K. Vicencio, T. Korras, K.A. Bordignon, and I. Gentilini. EnergyOptimal Path Planning for Six-Rotors on Multi-Target Missions. In Proc. IEEE/RSJ Int. Conf. Intel. Robots Syst., pages 2481-2487, 2015.

[12] D. Gandolfo, L. Salinas, A. Brandão, and J. Toibero. Stable PathFollowing Control for a Quadrotor Helicopter Considering Energy Consumption. IEEE Trans. Contr. Syst. Tech., 25(4):1423-1430, 2017.

[13] F. Morbidi, R. Cano, and D. Lara. Minimum-Energy Path Generation for a Quadrotor UAV. In Proc. IEEE Int. Conf. Robot. Automat., pages 1492-1498, 2016.

[14] M. Ryll, D. Bicego, and A. Franchi. Modeling and Control of FASTHex: a Fully-Actuated by Synchronized-Tilting Hexarotor. In Proc. IEEE/RSJ Int. Conf. Intel. Robots Syst., pages 1689-1694, 2016.

[15] G. Michieletto, M. Ryll, and A. Franchi. Fundamental Actuation Properties of Multirotors: Force-Moment Decoupling and Fail-Safe Robustness. IEEE Trans. Robot., 34(3):702-715, 2018.

[16] D. Brescianini and R. D'Andrea. Computationally Efficient Trajectory Generation for Fully Actuated Multirotor Vehicles. IEEE Trans. Robot., 34(3):555-571, 2018.

[17] S. Park et al. ODAR: Aerial Manipulation Platform Enabling OmniDirectional Wrench Generation. IEEE/ASME Trans. Mechatronics, 2018, in press. DOI: 10.1109/TMECH.2018.2848255.

[18] M. Ryll et al. 6D Physical Interaction with a Fully Actuated Aerial Robot. In Proc. IEEE Int. Conf. Robot. Automat., pages 5190-5195, 2017.

[19] TOMLAB Optimization, PROPT. [web]: http://tomdyn.com.

[20] J.I. Giribet, R.S. Sanchez-Pena, and A.S. Ghersin. Analysis and Design of a Tilted Rotor Hexacopter for Fault Tolerance. IEEE Trans. Aero. Elec. Sys., 52(4):1555-1567, 2016.

[21] R. Mahony, V. Kumar, and P. Corke. Multirotor Aerial Vehicles: Modeling, Estimation, and Control of Quadrotor. IEEE Rob. Autom. Mag., 19(3):20-32, 2012.

[22] D. Kirk. Optimal Control Theory: An Introduction. Dover Pub., 2004.

[23] D. Mellinger and V. Kumar. Minimum Snap Trajectory Generation and Control for Quadrotors. In Proc. IEEE Int. Conf. Robot. Automat., pages 2520-2525, 2011. 